\title{
Correction to: Flat coset decompositions of skew lattices
}

\author{
João Pita Costa ${ }^{1} \cdot$ Karin Cvetko-Vah²
}

Received: 19 June 2019 / Accepted: 22 July 2019 / Published online: 12 August 2019

(c) Springer Science+Business Media, LLC, part of Springer Nature 2019

\section{Correction to: Semigroup Forum (2016) 92:361-376 https://doi.org/10.1007/s00233-015-9739-8}

\begin{abstract}
In this corrigendum, we correct the statement and the proof of Proposition
\end{abstract} 4.11, where the word "lower" is stated instead of "upper," and vice versa.

Proposition 1 (Proposition 4.11) Let $S$ be a skew lattice such that $S / \mathcal{D}$ is a distributive lattice.

(i) Let $S$ be upper symmetric. Then, $S$ is upper cancellative, if and only if $M \vee x \vee$ $M=M \vee x^{\prime} \vee M \Leftrightarrow B \vee x \vee B=B \vee x^{\prime} \vee B$ holds for all skew diamonds $\{J>A, B>M\}$ in $S$ and all $x, x^{\prime} \in A$.

(ii) Let $S$ be lower symmetric. Then, $S$ is lower cancellative, if and only if $B \wedge x \wedge B=$ $B \wedge x^{\prime} \wedge B \Leftrightarrow J \wedge x \wedge J=J \wedge x^{\prime} \wedge J$ holdsfor all skew diamonds $\{J>A, B>M\}$ in $S$ and all $x, x^{\prime} \in A$.

Proof (i). A skew lattice is upper cancellative if and only if it is upper symmetric and simply cancellative. Assume first that $S$ is not upper cancellative, i.e., it is not simply cancellative. By a result of [5], it follows that $S$ contains a subalgebra $S^{\prime}$ isomorphic to $\mathbf{N C}_{5}$, given by the diagram below:

Communicated by Victoria Gould.

The original article can be found online at https://doi.org/10.1007/s00233-015-9739-8.

$凶 \quad$ Karin Cvetko-Vah

karin.cvetko@fmf.uni-lj.si

João Pita Costa

joao.pitacosta@ijs.si

1 Institut Jožef Stefan, Jamova Cesta 39, 1000 Ljubljana, Slovenia

2 Faculty of Mathematics and Physics, University of Ljubljana, Jadranska 19, 1000 Ljubljana, Slovenia 


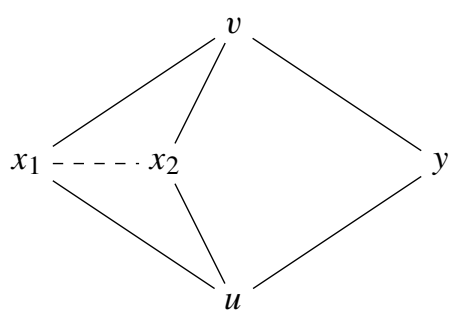

In $\mathbf{N C}_{5}$ operations on $\left\{x_{1}, x_{2}\right\}$ are defined in one of the following ways: either $x_{i} \wedge x_{j}=$ $x_{j}$ and $x_{i} \vee x_{j}=x_{i}$ which yields a right-handed structure; or $x_{i} \wedge x_{j}=x_{i}$ and $x_{i} \vee x_{j}=x_{j}$ yielding a left-handed structure. Let $A, B, M$ and $J$ denote the $\mathcal{D}$-classes of elements $x_{1}, y, u$ and $v$ in $S$, respectively. Note that $x_{2} \in A$. Since $x_{1}$ and $x_{2}$ are both contained in the image of $u$ in $A$, they cannot lie in the same coset of $M$ in $A$, i.e., we obtain $M \vee x_{1} \vee M \neq M \vee x_{2} \vee M$. On the other hand, the cosets $B \vee x_{1} \vee B$ and $B \vee x_{2} \vee B$ both contain $v$ (because $y \vee x_{1} \vee y=v=y \vee x_{2} \vee y$ ) and hence coincide by Theorem 2.4. Therefore, the implication $B \vee x_{1} \vee B=B \vee x_{2} \vee B \Rightarrow M \vee x_{1} \vee M=M \vee x_{2} \vee M$ fails.

Assume next that $S$ is upper cancellative and let $\{J>A, B>M\}$ be a skew diamond in $S$. Given any $x, x^{\prime} \in A$, the direct implication $M \vee x \vee M=M \vee x^{\prime} \vee M \Rightarrow$ $B \vee x \vee B=B \vee x^{\prime} \vee B$ holds by Remark 4.8. Assume that there exist $x_{1}, x_{2} \in A$ such that $B \vee x_{1} \vee B=B \vee x_{2} \vee B$, but $M \vee x_{1} \vee M \neq M \vee x_{2} \vee M$. Take any $m \in M$ and consider $a_{1}=m \vee x_{1} \vee m, a_{2}=m \vee x_{2} \vee m$. Note that $a_{1}, a_{2} \in A, m<a_{1}$ and $m<a_{2}$. Take $b \in B$ such that $b>m$. Since $m<a_{1}$ and $m<b$, it follows that $a_{1} \wedge b=m=b \wedge a_{1}$, and likewise $a_{2} \wedge b=m=b \wedge a_{2}$. By upper symmetry, $a_{1} \vee b=$ $b \vee a_{1}$ and $a_{2} \vee b=b \vee a_{2}$ follow. Denote $j_{1}=a_{1} \vee b$ and $j_{2}=a_{2} \vee b$. The assumption $B \vee x_{1} \vee B=B \vee x_{2} \vee B$ together with $m<b$ implies $b \vee a_{1} \vee b=b \vee m \vee x_{1} \vee m \vee b=$ $b \vee x_{1} \vee b=b \vee x_{2} \vee b=b \vee m \vee x_{2} \vee m \vee b=b \vee a_{2} \vee b$. It follows that $j_{1}=j_{2}$, and the set $S^{\prime}=\left\{m, a_{1}, a_{2}, b, j_{1}\right\}$ forms a subalgebra of $S$, given by the following diagram:

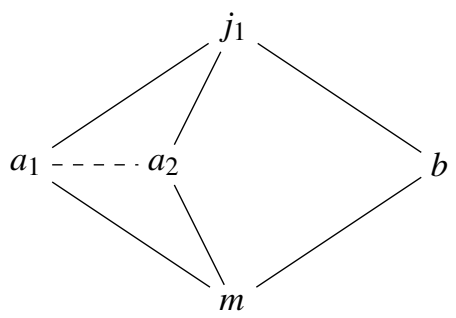

(Again, with the operations on $\left\{a_{1}, a_{2}\right\}$ defined either in a right-handed or in a lefthanded fashion.) Subalgebra $S^{\prime}$ is isomorphic to $\mathbf{N C}_{5}$. By a result of [5], it follows that $S$ is not simply cancellative and thus not upper cancellative, which is a contradiction.

The proof of (ii) is similar.

Publisher's Note Springer Nature remains neutral with regard to jurisdictional claims in published maps and institutional affiliations. 